\title{
Rare Decays of Heavy Mesons
}

\author{
Gaia LANFRANCHI* \\ (on behalf of the LHCb collaboration) \\ Laboratori Nazionali di Frascati, INFN \\ E-mail: Gaia.Lanfranchi@lnf.infn.it
}

Rare decays of heavy mesons provide some of the most promising approaches for testing the Standard Model with high level of accuracy. In this paper, the status and prospects in the field are reviewed, with a focus on the measurements performed by the LHCb, CMS and ATLAS collaborations at the Large Hadron Collider at CERN.

XXVII International Symposium on Lepton Photon Interactions at High Energies 17-22 August 2015

Ljubljana, Slovenia

${ }^{*}$ Speaker. 


\section{Introduction}

The Standard Model (SM) of particle physics cannot be the ultimate theory, as it is incomplete and contains too many free parameters, such as the fermion masses and the quark mixing angles. The pattern of these parameters could be governed by a hidden mechanism yet to be discovered, and so the SM would be a low-energy effective theory of a more fundamental theory that would be superseded by a higher energy scale, expected in the $\mathrm{TeV}$ region and accessible at the Large Hadron Collider (LHC). This would imply new symmetries, particles, dynamics, and flavor structure (New Physics, NP) that can be discovered either directly or indirectly.

The flavour, $C P$, and chirality structure of new interactions can be probed by measuring branching fractions, angular distributions and $C P$ asymmetries in decays of strange, charm, and beauty hadrons. Notably weak flavour-changing neutral current (FCNC) decays are very sensitive to contributions from heavy physics beyond the SM as they are both Cabibbo-Kobayashi-Maskawa (CKM) and loop-suppressed.

A wealth of new measurements of rare $b$ - and $c$-hadron decay processes have been performed by the ATLAS, CMS and LHCb experiments at the LHC and from the legacy datasets of the BaBar and Belle experiments. In the following paragraphs a review of the most recent results in the field of rare decays of heavy mesons is presented.

\section{Rare $b$-hadron decays}

Rare FCNC decays of $b$-hadrons are described by the weak effective Hamiltonian:

$$
H_{\mathrm{eff}}=-\frac{4 G_{F}}{\sqrt{2}} V_{t b} V_{t q}^{*} \frac{e^{2}}{16 \pi^{2}} \sum_{i}\left(C_{i} Q_{i}+C_{i}^{\prime} Q_{i}^{\prime}\right)+\text { h.c. }
$$

where $G_{F}$ is the Fermi constant and $V_{\mathrm{ij}}$ are CKM matrix elements. The $C_{i}(\mu)$ are Wilson coefficients corresponding to local operators with different Lorentz structure, $O_{i}(\mu)$. The operators and their Wilson coefficients are evaluated at the renormalisation scale $\mu$. Among the dimensionsix operators contributing to these transitions, the operators most sensitive to new physics effects are:

$$
\begin{array}{ll}
Q_{7}^{q\left({ }^{\prime}\right)}=\frac{m_{b}}{e}\left(\bar{q} \sigma_{\mu v} P_{R(L)} b\right) F^{\mu v} & Q_{8}^{q^{(\prime)}}=\frac{m_{b}}{e}\left(\bar{q} \sigma_{\mu v} P_{R(L)} T^{a} b\right) G^{a \mu v} \\
Q_{9}^{q\left({ }^{\prime}\right)}=\left(\bar{q} \gamma_{\mu} P_{L(R)} b\right)\left(\bar{l} \gamma^{\mu} l\right) & Q_{10}^{\left.q^{\prime}\right)}=\left(\bar{q} \gamma_{\mu} P_{L(R)} b\right)\left(\bar{l} \gamma^{\mu} \gamma_{5} l\right) \\
Q_{S}^{q\left({ }^{\prime}\right)}=\left(\bar{q} P_{L(R)} b\right)(\bar{l} l) & Q_{P}^{\left.q()^{\prime}\right)}=\left(\bar{q} P_{L(R)} b\right)\left(\bar{l} \gamma_{5} l\right) \\
Q_{L(R)}^{q}=\left(\bar{q} \gamma_{\mu} P_{L(R)} b\right)\left(\bar{v} \gamma^{\mu} P_{L} v\right) . &
\end{array}
$$

Here, $P_{L, R}$ denote left and right-hand chirality projections and $F^{\mu v}$ and $G^{a \mu v}$ are the electromagnetic and chromomagnetic field strength tensors, respectively. The electromagnetic and chromomagnetic dipole operators $Q_{7}^{\left({ }^{\prime}\right)}$ and $Q_{8}^{\left({ }^{\prime}\right)}$ contribute to radiative and semileptonic decays. The semileptonic operators $Q_{10, S, P}^{\left({ }^{\prime}\right)}$ contribute to leptonic and semileptonic decays, the operators $Q_{9}^{\left({ }^{\prime}\right)}$ only to semileptonic decays, and the operators $Q_{L, R}$ to decays with neutrinos in the final state. 


\section{Leptonic decays}

The important role of the branching fraction of the $B_{s}^{0} \rightarrow \mu^{+} \mu^{-}$and $B^{0} \rightarrow \mu^{+} \mu^{-}$decays in models with extended Higgs sector has been widely discussed in the literature. In fact, the axial contribution to the decays $B_{s}^{0} \rightarrow \mu^{+} \mu^{-}$and $B^{0} \rightarrow \mu^{+} \mu^{-}$is suppressed by the helicity factor $m_{\mu}^{2} / M_{B_{s, d}}^{2}$ and this renders these decays sensitive to physics with new scalar or pseudoscalar interactions and suitable to probe models with an extended Higgs sector. Practically all weakly coupled extensions of the Standard Model contain extra Higgs multiplets, which puts $B R\left(B_{s}^{0} \rightarrow \mu^{+} \mu^{-}\right)$and $B R\left(B^{0} \rightarrow \mu^{+} \mu^{-}\right)$at the center stage of indirect NP searches.

Several extensions of the SM predict enhancements to the branching fractions for these rare decays. In supersymmetric models with non-universal Higgs masses [1] and in specific models containing leptoquarks [2], the $B_{s}^{0} \rightarrow \mu^{+} \mu^{-}$and $B^{0} \rightarrow \mu^{+} \mu^{-}$branching fractions can be enhanced. In the minimal supersymmetric extension of the SM, the rates are strongly enhanced at large values of $\tan \beta$, which is the ratio of the two vacuum expectation values of the two Higgs boson doublets [3, 4]. However, in most models of New Physics, the decay rates can also be suppressed for specific choices of model parameters [5].

The branching fractions of these two decays, accounting for NLO electroweak corrections [6] and NNLO QCD corrections [7] are reliably calculated in the SM [8]. Their values are $B R\left(B_{s}^{0} \rightarrow\right.$ $\left.\mu^{+} \mu^{-}\right)=(3.66 \pm 0.23) \cdot 10^{-9}$ and $B R\left(B^{0} \rightarrow \mu^{+} \mu^{-}\right)=(1.06 \pm 0.09) \cdot 10^{-10}$. The uncertainties on the SM values are now dominated by our knowledge of the CKM matrix elements and the $B^{0}$ and $B_{s}^{0}$ decay constants $\left(f_{B^{0}}\right.$ and $\left.f_{B_{s}^{0}}\right)$, determined by Lattice QCD calculations [9]. A difference in the observed branching fractions with respect to the predictions would provide a direction in which the SM should be extended.

Before the LHC started operating, no evidence for either decay mode had been found. Upper limits on the branching fractions were an order of magnitude above the SM predictions. In $2013 \mathrm{LHCb}$ published the first evidence of the $B_{s}^{0} \rightarrow \mu^{+} \mu^{-}$decay based on $1.0 \mathrm{fb}^{-1}$ and $1.1 \mathrm{fb}^{-1}$ collected at $\sqrt{s}=7 \mathrm{TeV}$ and $\sqrt{s}=8 \mathrm{TeV}$, respectively [10]. Recently the CMS and LHCb collaborations have performed a joint analysis of the data collected during Run I [11] obtaining a statistical significance of $6.2 \sigma$ for the $B_{s}^{0}$ mode and $3.0 \sigma$ for the $B^{0}$ mode.

The combined fit leads to the measurements $B R\left(B_{s}^{0} \rightarrow \mu^{+} \mu^{-}\right)=\left(2.8_{-0.6}^{+0.7}\right) \cdot 10^{-9}$ and $B R\left(B^{0} \rightarrow\right.$ $\left.\mu^{+} \mu^{-}\right)=\left(3.9_{-1.4}^{+1.6}\right) \cdot 10^{-10}$, where the uncertainties include both statistical and systematic sources, the latter contributing as $35 \%$ and $18 \%$ of the total uncertainty for the $B_{s}^{0}$ and $B^{0}$ signals, respectively. The fit for the ratios of the branching fractions relative to their SM predictions yields $S_{\mathrm{SM}}^{B_{s}^{0}}=\left(0.76_{-0.18}^{+0.20}\right)$ and $S_{\mathrm{SM}}^{B^{0}}=\left(3.7_{-1.4}^{+1.6}\right)$. The measurements are compatible with the SM branching fractions of the $B_{s}^{0} \rightarrow \mu^{+} \mu^{-}$and $B^{0} \rightarrow \mu^{+} \mu^{-}$decays at the $1.2 \sigma$ and $2.2 \sigma$ level, respectively.

This result concludes a search that started more than three decades ago (see Fig. 1), and starts the era of precision measurements of the properties of this decay. The current precision on the $B R\left(B_{s}^{0} \rightarrow \mu^{+} \mu^{-}\right)$allows us to put constraints of the scale $\Lambda$ of new physics, $\Lambda>50 \mathrm{TeV}$ for generic couplings and $\Lambda>0.6 \mathrm{TeV}$ in case of Minimal Flavour Violation (MFV) loop. It is worth noting that the current accuracy reached on the Higgs couplings brings a similar sensitivity to the NP mass scale, $\Lambda>0.5-1.0 \mathrm{TeV}$ [12]. 


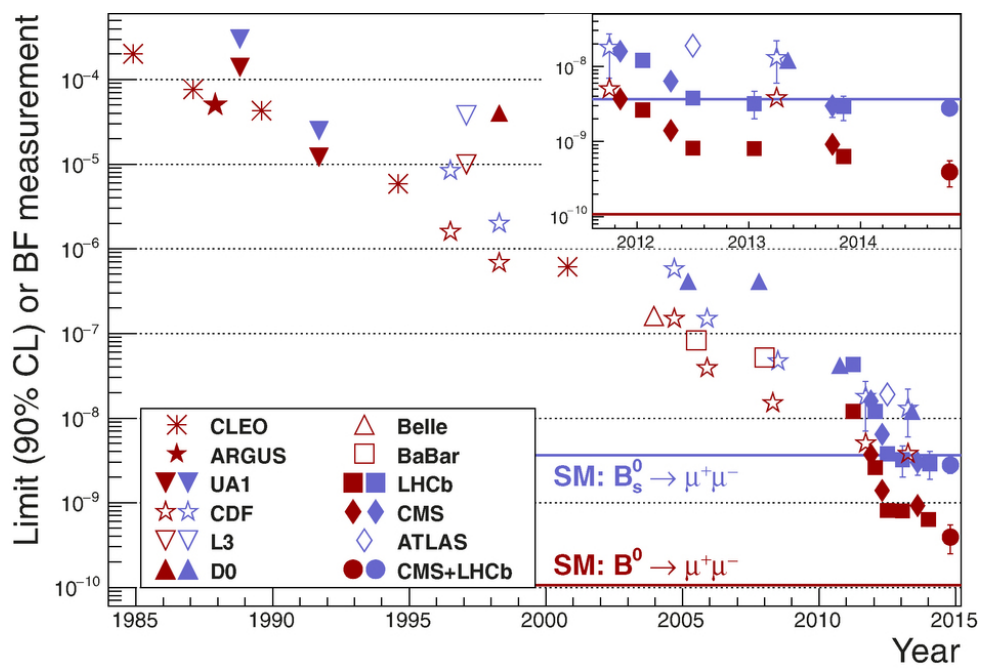

Figure 1: Search for the $B_{s}^{0} \rightarrow \mu^{+} \mu^{-}$and $B^{0} \rightarrow \mu^{+} \mu^{-}$decays, reported by 11 experiments spanning more than three decades, and by the present results. Markers without error bars denote upper limits on the branching fractions at $90 \%$ confidence level, while measurements are denoted with error bars delimiting $68 \%$ confidence intervals. The solid horizontal lines represent the SM predictions for the $B_{s}^{0} \rightarrow \mu^{+} \mu^{-}$and $B^{0} \rightarrow \mu^{+} \mu^{-}$ branching fractions; the blue (red) lines and markers relate to the $B_{s}^{0} \rightarrow \mu^{+} \mu^{-}\left(B^{0} \rightarrow \mu^{+} \mu^{-}\right)$decay. Figure from Ref. [11] and references therein.

\section{Exclusive semi-leptonic decays}

In the SM, the electroweak penguin decays $b \rightarrow s(d) \ell^{+} \ell^{-}$, where $\ell=\mu, e, \tau$, are only induced at the one-loop level, leading to small branching fractions and thus a rather high sensitivity to contributions from New Physics beyond the SM.

In particular, the rare decay $B^{0} \rightarrow K^{* 0} \mu^{+} \mu^{-}$is a $b \rightarrow s$ FCNC decay which is mediated by electroweak box and penguin diagrams in the SM. It can be a highly sensitive probe for new righthanded currents and new scalar and pseudoscalar couplings. These NP contributions can be probed by studying the angular distributions of the $B^{0}$ daughter particles.

The ATLAS, CMS and LHCb experiments have all performed measurements of the $B^{0} \rightarrow$ $K^{0 *}\left(\rightarrow K^{+} \pi^{-}\right) \mu^{+} \mu^{-}$angular distributions using data collected in $2011[13,14,15]$. Due to the rarity of the decay and the small number of candidates that are reconstructed, the experiments did not simultaneously fit for all of the angular terms. ATLAS and CMS measured only the longitudinal polarisation fraction of the $K^{* 0}, F_{\mathrm{L}}$, and the forward-backward asymmetry of the dimuon system, $A_{\mathrm{FB}}$. The LHCb experiment also measured the asymmetry between the two transverse $K^{* 0}$ polarisations, which is particularly sensitive to the handedness of the photon polarisation. All of these measurements were consistent with SM expectations.

The LHCb experiment also made first measurements of two new observables [16], $P_{4,5}^{\prime}$ which are free from form-factor uncertainties at leading order [17]. Interestingly, a large local-discrepancy of $3.7 \sigma$ has been seen between the measurement and the SM prediction in the dimuon invariant mass squared range $4.3<q^{2}<8.68 \mathrm{GeV}^{2} / \mathrm{c}^{4}$ for $P_{5}^{\prime}$. Recently this discrepancy has been confirmed by LHCb using the full Run I dataset, $3 \mathrm{fb}^{-1}$, and performing a simultaneous fit of all the angular 
distributions [18]. The results of the observables $F_{\mathrm{L}}, A_{\mathrm{FB}}$ and $P_{5}^{\prime}$ are shown in Fig. 2 and Fig. 3 respectively, with the SM prediction from [19, 20] and [21]. Both $F_{L}$ and $A_{F B}$ agree with SM predictions, while the $P_{5}^{\prime}$ observable is measured to be above the SM prediction [21] for $q^{2}$ between $4 \mathrm{GeV}^{2} / c^{4}$ and $8 \mathrm{GeV}^{2} / \mathrm{c}^{4}$ (see Fig. 3). This result is compatible with the previous measurement [16] and corresponds to a deviation of $2.9 \sigma$ in the $q^{2}$ bins, [4.0-6.0] $\mathrm{GeV}^{2} / \mathrm{c}^{4}$ and [6.0-8.0] $\mathrm{GeV}^{2} / \mathrm{c}^{4}$. The other observables, $S_{3-9}$, are in good agreement with the SM prediction [18].
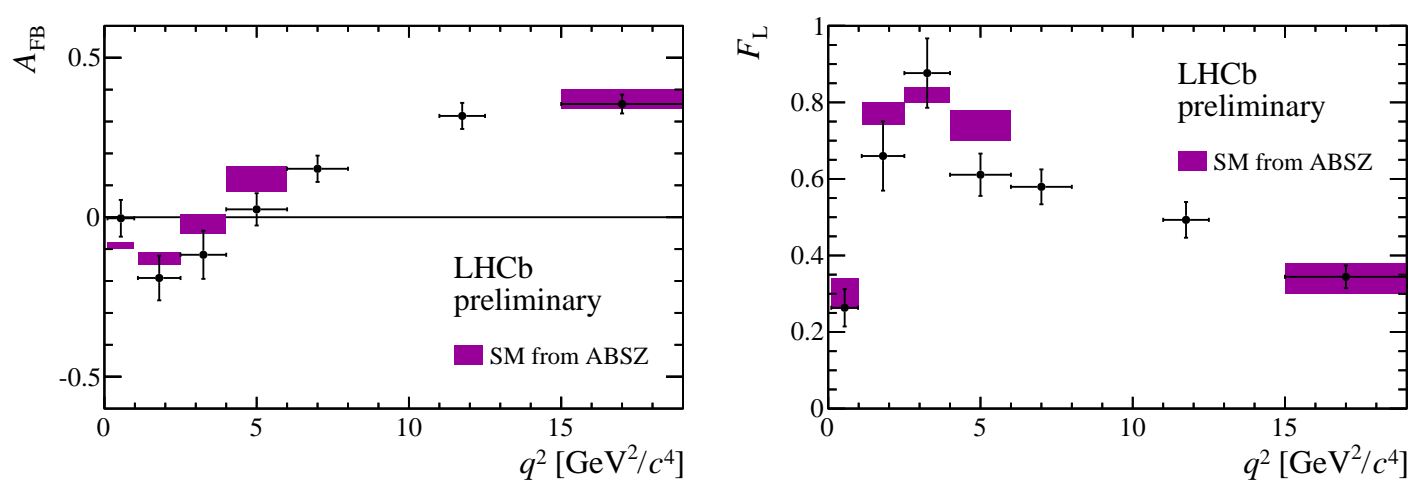

Figure 2: $A_{\mathrm{FB}}$ and $F_{\mathrm{L}}$ in bins of $q^{2}$. The shaded boxes show the SM prediction taken from Ref. [20].

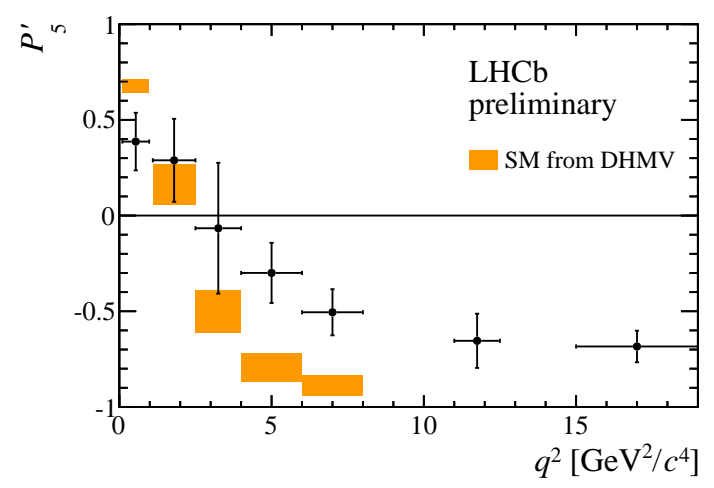

Figure 3: The observable $P_{5}^{\prime}$ in bins of $q^{2}$. The shaded boxes show the SM prediction taken from Ref. [21].

Recently the CMS collaboration published an update of the analysis of the $B^{0} \rightarrow K^{* 0} \mu^{+} \mu^{-}$ decay, measuring the forward-backward asymmetry of the muons, the $K^{* 0}$ longitudinal polarization fraction, and the differential branching fraction as a function of $q^{2}$ [22]. The results are shown in Fig. 4. This update is based on $20 \mathrm{fb}^{-1}$ of data collected in 2012 at $\sqrt{s}=8 \mathrm{TeV}$. The measurements are among the most precise to date and are in good agreement with the SM predictions. The data collected in the coming years will allow CMS to perform the full angular analysis of the $B^{0} \rightarrow K^{* 0} \mu^{+} \mu^{-}$decays and measure the angular distribution of the $P_{5}^{\prime}$ variable.

Branching ratio measurements of $B^{0} \rightarrow K^{* 0} \mu^{+} \mu^{-}, B^{0} \rightarrow K_{s} \mu^{+} \mu^{-}$, and $B^{+} \rightarrow K^{*+} \mu^{+} \mu^{-}$ decays published by $\mathrm{LHCb}$ [26] seem to be too low compared to the SM predictions when using 

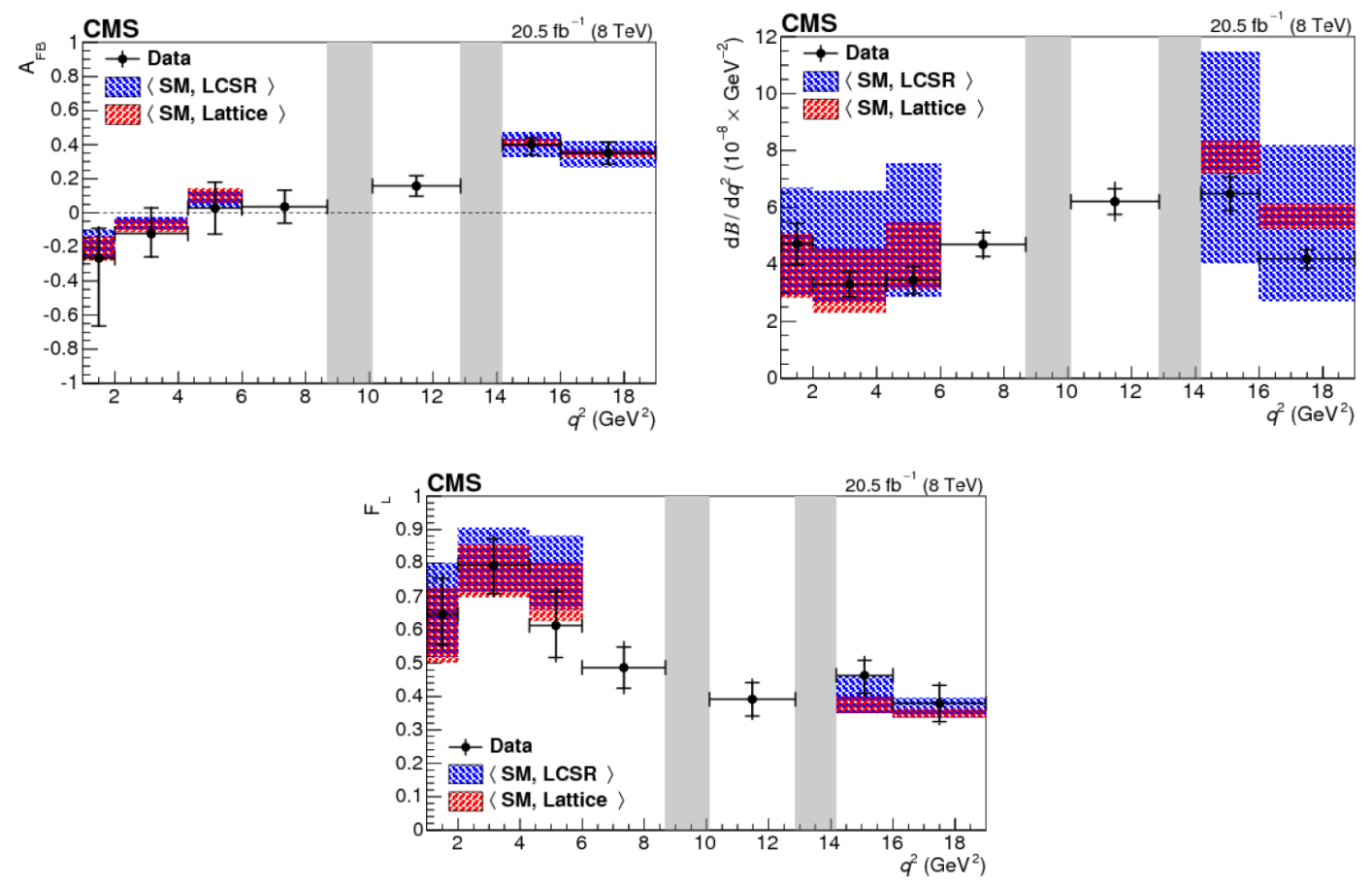

Figure 4: Measured values of $F_{L}, A_{\mathrm{FB}}$, and $d B / d q^{2}$ vs $q^{2}$ for $B^{0} \rightarrow K^{* 0} \mu^{+} \mu^{-}$. The statistical uncertainty is shown by the inner vertical bars, while the outer vertical bars give the total uncertainty. The horizontal bars show the bin widths. The vertical shaded regions correspond to the $J / \psi$ and $\Psi(2 S)$ resonances. The blue and red shaded regions show two Standard Model predictions: the light-cone sum rules (LCSR) calculation is made at low $q^{2}$ [23] and is extrapolated to high $q^{2}$ [24]. The lattice gauge (Lattice) calculation of the form factors is from Ref. [25].

state-of-the art form factors from lattice QCD or light-cone sum rules (LCSR) [27, 28, 29, 30]. Recently the differential branching fraction and angular analysis of the $B_{S} \rightarrow \phi \mu^{+} \mu^{-}$decay has been updated by the LHCb collaboration using the full Run I dataset [31]. Results of the angular analysis are found to be consistent with the Standard Model. The differential branching fraction is found instead to be $3.3 \sigma$ below the SM predictions in the range $1<q^{2}<6 \mathrm{GeV}^{2} / \mathrm{c}^{4}$, where precise theoretical calculations are available (Fig. 5).

In 2014, another tension with the SM has been observed by LHCb, namely a suppression of the ratio $R_{K}$ of $B^{+} \rightarrow K^{+} \mu^{+} \mu^{-}$and $B^{+} \rightarrow K^{+} e^{+} e^{-}$branching fractions at low di-lepton invariant mass [32]. In the SM this ratio is expected to differ from unity only due to tiny Higgs penguin contributions and difference of phase space, $R_{K}^{\mathrm{SM}}=1+o\left(m_{\mu}^{2} / m_{b}^{2}\right)=1.0003 \pm 0.0001$ [8]. The impact of radiative corrections in the ratio has been evaluated employing the cuts on the dilepton invariant mass squared and the reconstructed $B$-meson mass presently applied by the LHCb collaboration and does not exceed a few $\%$ [33]. The LHCb result, $R_{K}=0.745_{-0.074}^{+0.090}$ (stat) \pm 0.036 (syst), is currently $2.6 \sigma$ below the SM prediction.

Several attempts have been made to understand these anomalies by performing global fits to the data from the LHC experiments, CDF and the B factories. Reference [34] performed a global 


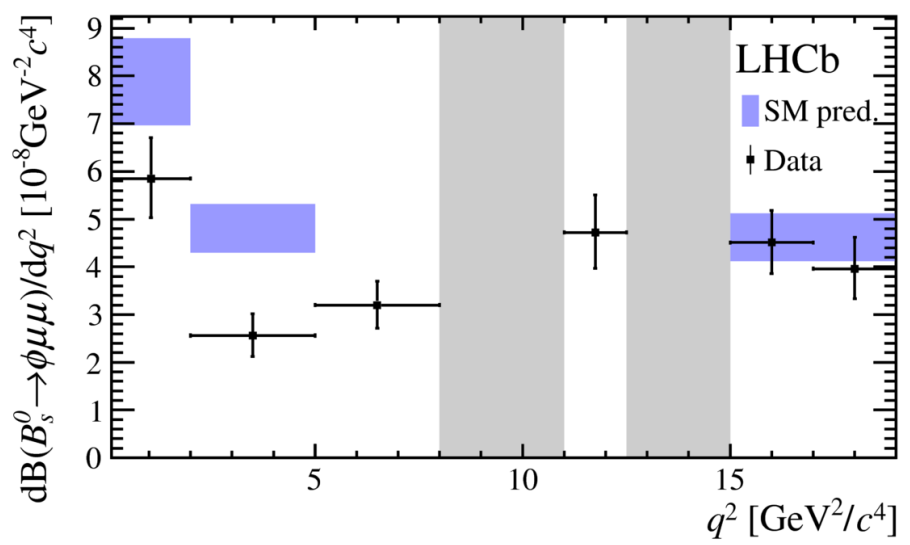

Figure 5: Differential branching fraction of the decay $B_{S}^{0} \rightarrow \phi \mu^{+} \mu^{-}$, overlaid with SM predictions [19, 20] indicated by blue shaded boxes. The vetoes excluding the charmonium resonances are indicated by grey areas.

fit to 88 measurements of 76 different observables from six experiments, including $B^{0} \rightarrow K^{*} \mu^{+} \mu^{-}$, angular observables and branching fractions of $B^{0} \rightarrow K_{S} \mu^{+} \mu^{-}, B^{+} \rightarrow K^{(*)+} \mu^{+} \mu^{-}, B_{s} \rightarrow \phi \mu^{+} \mu^{-}$, $B^{0} \rightarrow K^{*} \gamma, B^{0} \rightarrow X_{s} \gamma, B_{s} \rightarrow \mu^{+} \mu^{-}$.

The data are best described by a model in which a new vector current destructively interferes with the SM contributions $[35,36,37]$. The effect is especially pronounced at low $q^{2}$ due to additional interference with the virtual photon contribution in the SM. A new vector current is best explained in models that introduce a $Z^{\prime}$ boson with flavour violating couplings [38]. This model would also predict $R_{K} \simeq 0.75$, close to what is measured in data. Other $Z^{\prime}$ models have been suggested in the literature as well $[39,40,41]$. However, it is also possible that the discrepancies observed in the angular analysis of the $B^{0} \rightarrow K^{* 0} \mu^{+} \mu^{-}$could be explained by an underestimate of the uncertainty on the SM prediction, coming from the treatment of the form-factors [42] or from our understanding of $c \bar{c}$ contributions to the decay [43].

Future improved measurements of $B^{0} \rightarrow K^{* 0} \mu^{+} \mu^{-}$angular observables will allow for a significantly more precise determination of the Wilson coefficients. In this respect, an important role is played by angular $C P$ asymmetries. These will allow to determine the imaginary parts of the Wilson coefficients, which are still constrained very weakly.

Recently LHCb published the results related to the study of $b \rightarrow d \mu^{+} \mu^{-}$transitions in $B^{0} \rightarrow$ $\pi^{+} \mu^{+} \mu^{-}$decays [44]. The measured values of the differential branching fraction are shown in Fig. 6. The branching fraction agrees with SM predictions from Refs. [45, 46], although agreement in the lowest $-q^{2}$ bin is only achieved when contributions from low- $q^{2}$ resonances are taken into account [46]. The total branching fraction is computed from the integral over the measured bins multiplied by a scaling factor to account for the regions of $q^{2}$ not measured in this analysis and is found to be in agreement with the SM predictions. The CP asymmetry of the decay has been measured and is consistent with a recent SM prediction [46]. The values for the CKM matrix elements $\left|V_{t d}\right|$ and $\left|V_{t s}\right|$ and the ratio $\left|V_{t d} / V_{t s}\right|$ have also been determined using the $B^{+} \rightarrow \pi^{+} \mu^{+} \mu^{-}$ and the $B^{+} \rightarrow K^{+} \mu^{+} \mu^{-}$decays, and are in agreement with previous measurements [47]. 


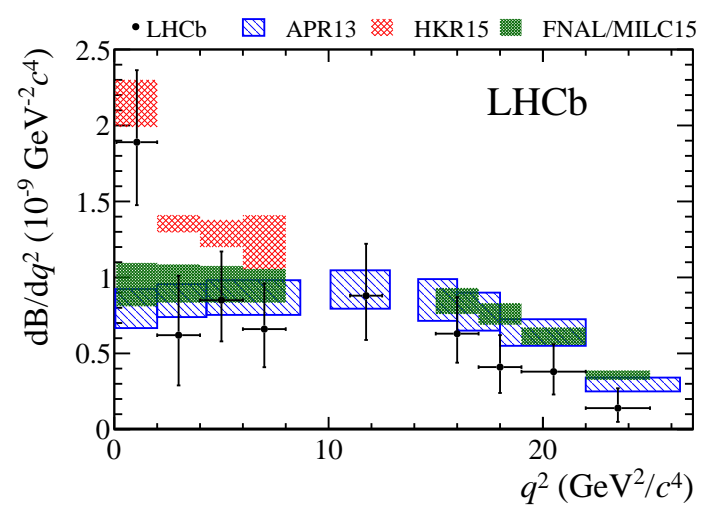

Figure 6: The differential branching fraction of $B^{+} \rightarrow \pi^{+} \mu^{+} \mu^{-}$in bins of dilepton invariant mass squared, $q^{2}$, compared to SM predictions taken from Refs. [45] (APR13), [46] (HKR15) and from lattice QCD calculations [48] (FNAL/MILC15).

\section{The hidden sector}

We expected TeV-scale NP with sizable couplings to solve the hierarchy problem and since it is easy to obtain dark matter out of it, there was no need for a light hidden sector. Now, motivated by the current lack of evidence for a dark matter particle candidate, interest has been rekindled in hidden-sector theories [49]-[56]. These theories postulate that dark matter particles interact feebly with all the known particles and, hence, have thus far escaped detection. Hidden sector particles are singlet states under the SM gauge interactions. Coupling between the SM and hidden-sector particles may arise via mixing of the hidden-sector field with a SM portal field. Any SM field whose particle does not carry electromagnetic charge (the Higgs and the $Z$ bosons, the photon and the neutrinos) could provide such a portal.

Many theories predict that $\mathrm{TeV}$-scale dark matter particles interact via $\mathrm{GeV}$-scale bosons [57, 58, 59]. Previous searches for such GeV-scale particles have been performed using large data samples from many types of experiments (see Ref.[60] for a summary). One class of models involving the scalar portal hypothesizes that such a field was responsible for an inflationary period in the early universe [61], and may have generated the baryon asymmetry observed today [62, 63]. The associated inflaton particle is expected to have a mass in the range $270-1800 \mathrm{MeV}$ [61]. Another class of models invokes the axial-vector portal in theories of dark matter that seek to address the cosmic ray anomalies, and to explain the suppression of $C P$ violation in strong interactions [64]. These theories postulate an additional fundamental symmetry, the spontaneous breaking of which results in a particle called the axion [65]. To couple the axion portal to a hidden sector containing a $\mathrm{TeV}$-scale dark matter particle, while also explaining the suppression of $C P$ violation in strong interactions, Ref.[66] proposes an axion with 360-800 $\mathrm{MeV}$ and an energy scale, $f$, at which the symmetry is broken, in the range $1-3 \mathrm{TeV}$. A broader range of mass and energy scale values is allowed in other dark matter scenarios involving axion(-like) states [67, 68, 69].

LHCb has published a search for a hidden-sector boson $\chi$ produced in the decay $B^{0} \rightarrow K^{* 0} \chi$ with $K^{* 0} \rightarrow K^{+} \pi^{-}$and $\chi \rightarrow \mu^{+} \mu^{-}$based on $3 \mathrm{fb}^{-1}$ [70]. No evidence for a signal is observed, and 
upper limits are placed on $B R\left(B^{0} \rightarrow K^{* 0} \chi\right) \times B R\left(\chi \rightarrow \mu^{+} \mu^{-}\right)$as a function of mass and lifetime of the $\chi$ boson. These limits are of the order of $10^{-9}$ for $\chi$ lifetimes less than $100 \mathrm{ps}$ and for $m_{\mu \mu}<1 \mathrm{GeV} / \mathrm{c}^{2}$. Figure 7 (left) shows exclusion regions for the DFSZ [71, 72] axion model of Ref. [68] set in the limit of large ratio of Higgs-doublet vacuum expectation values, $\tan \beta \geq 3$, for charged-Higgs masses of 1 and $10 \mathrm{TeV}$ and for two extreme cases, $B R(\chi \rightarrow$ hadrons $)=0$ and 0.99 . Figure 7 (right) shows exclusion regions for the inflaton model of Ref. [73], which only considers $m(\chi)<1 \mathrm{GeV}$. The branching fraction into hadrons is taken directly from Ref. [73] and, as in the axion model, is highly uncertain but this does not greatly affect the sensitivity of this search. Constraints are placed on the mixing angle between the Higgs and inflaton fields, $\theta$, which exclude the previously allowed region for $m_{\mu \mu}<1 \mathrm{GeV} / \mathrm{c}^{2}$.
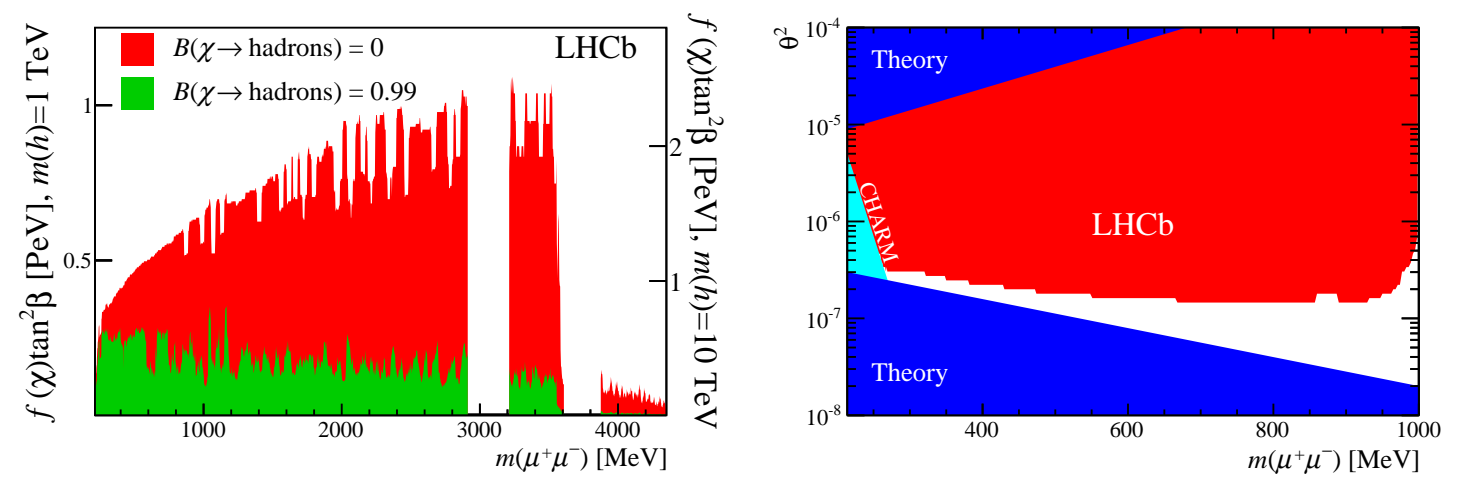

Figure 7: Exclusion regions at 95\% CL: (left) constraints on the axion model of Ref. [68]; (right) constraints on the inflaton model of Ref. [73]. The regions excluded by the theory [73] and by the CHARM experiment [74] are also shown.

\section{Conclusions}

A wealth of experimental results in the last few years are constraining New Physics (NP) contributions in rare decays of heavy mesons, in particular in $b \rightarrow s \ell^{+} \ell^{-}$transitions, with unprecedented level of precision. We did not observe so far any unambiguous deviation from SM predictions, hence either NP is very heavy or it mimics the SM in its flavor-breaking pattern. Another intriguing possibility is that New Physics is below the Fermi scale and couples very weakly to SM particles and escaped detection so far. The quest for NP in rare decays of heavy mesons will continue in the coming years and will nicely complement the constraints on NP coming from direct searches and from the study of the Higgs couplings. 


\section{References}

[1] J. R. Ellis, K. A. Olive, Y. Santoso et al., On $B_{s}^{0} \rightarrow \mu^{+} \mu^{-}$and cold matter scattering in the MSSM wth non-universal Higgs masses, JHEP 05063 (2006).

[2] S. Davidson and S. Descotes-Genon, Minimal Flavour Violation for Leptoquarks, JHEP 11, 073 (2010).

[3] S. R. Choudhury, A. S. Cornell, N. Gaur et al., Signatures of new Physics in dileptonic B-decays, Int. J. Mod. Phys. A 21, 2617 (2006).

[4] J. Parry, Lepton flavour violating Higgs Boson decays, $\tau \rightarrow \mu \gamma$ and $B_{(s)}^{0} \rightarrow \mu^{+} \mu^{-}$in the constrained MSSM+NR with large $\tan \beta$, Nucl. Phys. B 760, 38 (2007).

[5] J. R. Ellis, J. S. Lee, and A. Pilaftsis, B-meson observables in the maximally CP-violating MSSM with minimal flavor violation, Phys. Rev. D 76, 115011 (2007).

[6] C. Bobeth, M. Gorbahn, and E. Stamou, Electroweak Corrections to $B_{s d} \rightarrow \mu^{+} \mu^{-}$, Phys. Rev. D89 (2014), no. 3 034023, arXiv:1311.1348.

[7] T. Hermann, M. Misiak, and M. Steinhauser, Three-loop QCD corrections to $B_{s} \rightarrow \mu^{+} \mu^{-}$, JHEP 12 (2013) 097, arXiv:1311.1347.

[8] C. Bobeth et al. $B_{s, d} \rightarrow \ell^{+} \ell^{-}$in the Standard Model with reduced theoretical uncertainty, Phys. Rev. Lett. 112, 101801 (2014).

[9] S. Aoki et al., Review of lattice results concerning low-energy particle physics, Eur. Phys. J. C74 (2014), no. 9 2890, arXiv:1310.8555.

[10] R. Aaij et al. (LHCb collaboration), First evidence of the decay $B_{s}^{0} \rightarrow \mu^{+} \mu^{-}$, Phys. Rev. Lett. 110 (2013) 021801.

[11] LHCb and CMS collaborations, Observation of the rare $B_{s}^{0} \rightarrow \mu^{+} \mu^{-}$decay from the combined analysis of CMS and LHCb data, Nature 522 (2015) 68.

[12] M. Muhlleitner, Higgs Couplings and their Implications for New Physics Scales, arXiv:1506.03070v1 [hep-ph].

[13] ATLAS collaboration, Angular Analysis of $B^{0} \rightarrow K^{* 0} \mu^{+} \mu^{-}$with the ATLAS Experiment, ATLAS-CONF-2013-038 (2013).

[14] S. Chatrchyan et al. (CMS collaboration), Angular analysis and branching fraction measurement of the decay $B^{0} \rightarrow K^{* 0} \mu^{+} \mu^{-}$, Phys. Lett. , B 727 (2013) 77.

[15] R. Aaij et al. (LHCb collaboration), Differential branching fraction and angular analysis of the decay $B^{0} \rightarrow K^{* 0} \mu^{+} \mu^{-}$, JHEP, 08 (2013) 131.

[16] R. Aaij et al. (LHCb collaboration), Measurement of form-factor independent observables in the decay $B^{0} \rightarrow K^{* 0} \mu^{+} \mu^{-}$, Phys. Rev. Lett. 111 (2013) 191801.

[17] S. Descotes-Genonet al., Optimizing the basis of $B \rightarrow K^{*} \ell \ell$ observables in the full kinematic range JHEP 05 (2013) 137.

[18] R. Aaij et al. (LHCb collaboration), Angular analysis of the $B^{0} \rightarrow K^{0 *} \mu^{+} \mu^{-}$decay, LHCb-CONF-2015-002, cds.cern.ch/record/2002772.

[19] W. Altmannshofer and D. M. Straub, State of new physics in $b \rightarrow$ s transitions, Eur. Phys. J. C (2015) 75:382, arXiv:1411.3161. 
[20] A. Bharucha, D. M. Straub, and R. Zwicky, $B \rightarrow V l^{+} l^{-}$in the Standard Model from light cone sum rules, arXiv:1503.05534.

[21] S. Descotes-Genon et al., On the impact of power corrections in the prediction of $B \rightarrow K^{* 0} \mu^{+} \mu^{-}$ observables, JHEP 12 (2014) 125.

[22] CMS collaboration, Angular analysis of the decay BO to $K^{*} 0$ mu mu from pp collisions at sqrt $(s)=8$ TeV, arXiv:1507.08126 .

[23] A. Khodjamirian, T. Mannel, A. A. Pivovarov, and Y.-M. Wang, Charm-loop effect in $B \rightarrow K^{*} l^{+} l^{-}$ and $B \rightarrow K^{*} \gamma$, JHEP 09 (2010) 089.

[24] A. Khodjamirian, T. Mannel, and Y.-M. Wang, $B \rightarrow K \ell^{+} \ell^{-}$at large hadronic recoil, JHEP 02 (2013) 010.

[25] R. R. Horgan, Z. Liu, S. Meinel, and M. Wingate, Lattice QCD calculation of form factors describing the rare decays $B \rightarrow K^{*} \ell^{+} \ell^{-}$and $B_{S} \rightarrow \phi \ell^{+} \ell^{-}$, Phys. Rev. D89 (2014) 094501.

[26] R. Aaij et al. (LHCb collaboration), Differential branching fractions and isospin asymmetries of $B \rightarrow K^{* 0} \mu^{+} \mu^{-}$decays, JHEP 06 (2014) 133.

[27] P.M. Ferreira et al., Wrong sign and symmetric limits and non-decoupling in 2HDMs, JHEP 07 (2014) 067.

[28] C. Bobeth et al., The Decay $B \rightarrow K l^{+} l^{-}$at low hadronic recoil and model-independent $\Delta B=1$ constraints, JHEP 01 (2012) 107.

[29] C. Bouchard et al., Standard Model Predictions for $B \rightarrow K \ell^{+} \ell^{-}$BâE Ěs with Form Factors from Lattice QCD, PRL 111 (2013) 162002.

[30] R. R. Horgan, Zhaofeng Liu, Stefan Meinel, and Matthew Wingate, Calculation of $B^{0} \rightarrow K^{* 0} \mu^{+} \mu$ and $B_{s}^{0} \rightarrow \phi \mu^{+} \mu^{-}$observables using form factors from Lattice QCD, PRL 112 (2014) 212003.

[31] R. Aaij et al. (LHCb collaboration), Angular analysis and differential branching fraction of the decay $B_{s}^{0} \rightarrow \phi \mu^{+} \mu^{-}$, JHEP 09 (2015) 179.

[32] R. Aaij et al. (LHCb collaboration), Test of lepton universality using $B^{+} \rightarrow K^{+} \ell^{+} \ell^{-}$decays, Phys. Rev. Lett. 113 (2014), 151601.

[33] M. Bordone, G. Isidori, A. Pattori, On the Standard Model predictions for $R_{K}$ and $R_{K}^{*}$, arXiv:1605.07633 [hep-ph].

[34] W. Altmannshofer and D. M. Straub, Implications of $b \rightarrow s$ measurements, EPJC 8 (2015) 382.

[35] S. Descotes-Genon et al., Understanding the $B^{0} \rightarrow K^{* 0} \mu^{+} \mu^{-}$anomaly, Phys. Rev. D 88 (2013) 074002 .

[36] W. Altmannshofer and D. M. Straub, New physics in $B^{0} \rightarrow K^{* 0} \mu^{+} \mu^{-}$, Eur. Phys. J., C73 (2013) 2646.

[37] F. Beaujean, C. Bobeth and D. van Dyk, Comprehensive Bayesian Analysis of Rare (Semi)leptonic and Radiative B Decays, Eur. Phys. J., C74 (2014) 2897.

[38] R. Gauld and F. Goertz and U. Haisch, An explicit $Z^{\prime}$-boson explanation of the $B \rightarrow K^{*} \mu^{+} \mu^{-}$ anomaly, JHEP 01 (2014) 069.

[39] R. Gauld, F. Goertz, and U. Haisch, On minimal $Z^{\prime}$ explanations of the $B^{0} \rightarrow K^{* 0} \mu^{+} \mu^{-}$anomaly, Phys. Rev. D89 (2014) 015005, arXiv:1308.1959.

[40] A. J. Buras and J. Girrbach, Left-handed $Z^{\prime}$ and Z FCNC quark couplings facing new $b \rightarrow s \mu \mu$ data, JHEP 12 (2013) 009, arXiv:1309.2466. 
[41] A. J. Buras, F. De Fazio, and J. Girrbach, 331 models facing new $b \rightarrow s \mu^{+} \mu^{-}$data, JHEP 02 (2014) 112, arXiv:1311.6729.

[42] S. Jäger and J. Martin Camalich, On B $\rightarrow$ Vll at small dilepton invariant mass, power corrections, and new physics, JHEP 05 (2013) 043.

[43] J. Lyon J. and R. Zwicky, Resonances gone topsy turvy -the charm of QCD or new physics in $b \rightarrow s \ell^{+} \ell^{-}$, arXiv: 1406.0566 .

[44] R. Aaij et al. (LHCb collaboration), First measurement of the differential branching ratio and CP asymmetry of the $B^{+} \rightarrow \pi^{+} \mu^{-} \mu^{+}$decay, arXiv:1509.00414.

[45] A. Ali, A. Y. Parkhomenko, and A. V. Rusov, Precise calculation of the dilepton invariant-mass spectrum and the decay rate in $B^{ \pm} \rightarrow \pi^{ \pm} \mu^{+} \mu^{-}$in the SM, Phys. Rev. D89 (2014) 094021.

[46] C. Hambrock, A. Khodjamirian, and A. Rusov, Hadronic effects and observables in $B \rightarrow \pi \ell^{+} \ell^{-}$ decay at large recoil, Phys. Rev. D 92, 074020, arXiv:1506.07760.

[47] K.A. Olive et al. (Particle Data Group), Chin. Phys. C 38, 090001 (2014).

[48] J. A. Bailey et al., $B \rightarrow \pi \ell \ell$ form factors for new-physics searches from lattice $Q C D$, arXiv:1507.01618.

[49] R. Essig et al., Dark sectors and new, light, weakly-coupled particles, arXiv:1311.0029, prepared as part of the Community Summer Study 2013 (Snowmass).

[50] G. Weidenspointner et al., The sky distribution of positronium annihilation continuum emission measured with SPI/INTEGRAL, Astron. Astrophys. 450 (2006) 1012, arXiv:astro-ph/0601673.

[51] J. Chang et al., An excess of cosmic ray electrons at energies of 300-800 GeV, Nature 456 (2008) 362.

[52] O. Adriani et al. (PAMELA collaboration), An anomalous positron abundance in cosmic rays with energies 1.5-100 GeV, Nature $\mathbf{4 5 8}$ (2009) 607, arXiv:0810.4995.

[53] O. Adriani et al. (PAMELA collaboration), Cosmic-ray electron flux measured by the PAMELA experiment between 1 and 625 GeV, Phys. Rev. Lett. 106 (2011) 201101, arXiv:1103.2880.

[54] O. Adriani et al. (PAMELA collaboration), Cosmic-ray positron energy spectrum measured by PAMELA, Phys. Rev. Lett. 111 (2013) 081102, arXiv:1308.0133.

[55] M. Ackermann et al., (Fermi LAT collaboration ), Measurement of separate cosmic-ray electron and positron spectra with the Fermi Large Area Telescope, Phys. Rev. Lett. 108 (2012) 011103, arXiv:1109.0521.

[56] M. Aguilar et al. (AMS collaboration), Electron and positron fluxes in primary cosmic rays measured with the Alpha Magnetic Spectrometer on the International Space Station, Phys. Rev. Lett. 113 (2014) 121102.

[57] N. Arkani-Hamed, D. P. Finkbeiner, T. R. Slatyer, and N. Weiner, A theory of dark matter, Phys. Rev. D79 (2009) 015014, arXiv:0810.0713.

[58] M. Pospelov and A. Ritz, Astrophysical signatures of secluded dark matter, Phys. Lett. B671 (2009) 391, arXiv:0810.1502.

[59] C. Cheung, J. T. Ruderman, L.-T. Wang, and I. Yavin, Kinetic mixing as the origin of a light dark-gauge-group scale, Phys. Rev. D80 (2009) 035008, arXiv:0902.3246.

[60] S. Alekhin et al., A facility to Search for Hidden Particles at the CERN SPS: the SHiP physics case, arXiv:1504.04855. 
[61] F. Bezrukov and D. Gorbunov, Light inflaton hunter's guide, JHEP 05 (2010) 010, arXiv:0912.0390.

[62] M. P. Hertzberg and J. Karouby, Generating the observed baryon asymmetry from the inflaton field, Phys. Rev. D89 (2014) 063523, arXiv:1309.0010.

[63] M. P. Hertzberg and J. Karouby, Baryogenesis from the inflaton field, Phys. Lett. B737 (2014) 34, arXiv:1309.0007.

[64] R. D. Peccei, The strong CP problem and axions, Lect. Notes Phys. 741 (2008) 3, arXiv:hep-ph/0607268.

[65] R. D. Peccei and H. R. Quinn, CP conservation in the presence of pseudoparticles, Phys. Rev. Lett. 38 (1977) 1440.

[66] Y. Nomura and J. Thaler, Dark matter through the axion portal, Phys. Rev. D79 (2009) 075008, arXiv:0810.5397.

[67] J. Mardon, Y. Nomura, and J. Thaler, Cosmic signals from the hidden sector, Phys. Rev. D80 (2009) 035013, arXiv:0905.3749.

[68] M. Freytsis, Z. Ligeti, and J. Thaler, Constraining the axion portal with $B \rightarrow K \ell^{+} \ell^{-}$, Phys. Rev. D81 (2010) 034001, arXiv:0911.5355.

[69] D. Hooper and T. M. P. Tait, Neutralinos in an extension of the minimal supersymmetric standard model as the source of the PAMELA positron excess, Phys. Rev. D80 (2009) 055028, arXiv:0906.0362.

[70] R. Aaij et al. (LHCb collaboration), Search for hidden-sector bosons in $B^{0} \rightarrow K^{* 0} \mu^{+} \mu^{-}$decays, Phys. Rev. Lett. 115 (2015) 161802, arXiv: 1508.04094.

[71] M. Dine, W. Fischler, and M. Srednicki, A simple solution to the strong CP problem with a harmless axion, Phys. Lett. B104 (1981) 199.

[72] A. R. Zhitnitsky, On possible suppression of the axion hadron interactions, Sov. J. Nucl. Phys. 31 (1980) 260.

[73] F. Bezrukov and D. Gorbunov, Relic gravity waves and 7 keV dark matter from a GeV scale inflaton, Phys. Lett. B736 (2014) 494, arXiv:1403.4638.

[74] F. Bergsma et al. (CHARM collaboration), Search for axion like particle production in $400 \mathrm{GeV}$ proton-copper interactions, Phys. Lett. B157 (1985) 458. 